\title{
An Unusual Case of Herpes Simplex Meningitis after Inadvertent Puncture of the Subarachnoid Space
}

\author{
Richell Bastos Vale1, Bruno Vítor Martins Santiago ${ }^{2}$, José Abel de Almeida Neto', \\ Luiz Otávio Ribeiro de Lemos Felgueiras ${ }^{3}$, Karine Grillo de Freitas ${ }^{3}$, \\ Thiago dos Santos Ferreira1,3
}

${ }^{1}$ Department of Anesthesiology, Piedade Municipal Hospital, Rio de Janeiro, Brazil

${ }^{2}$ Department of Anesthesiology, Marcílio Dias Naval Hospital, Rio de Janeiro, Brazil

${ }^{3}$ Interleague Association of Anesthesiology of the State of Rio de Janeiro, Rio de Janeiro, Brazil

Email: *santiago.bruno@posgraduação.uerj.br

How to cite this paper: Vale, R.B., Santiago, B.V.M., de Almeida Neto, J.A., de Lemos Felgueiras, L.O.R., de Freitas, K.G. and dos Santos Ferreira, T. (2021) An Unusual Case of Herpes Simplex Meningitis after Inadvertent Puncture of the Subarachnoid Space. Case Reports in Clinical Medicine, 10, 232239.

https://doi.org/10.4236/crcm.2021.109030

Received: July 31, 2021

Accepted: August 31, 2021

Published: September 3, 2021

Copyright $\odot 2021$ by author(s) and Scientific Research Publishing Inc. This work is licensed under the Creative Commons Attribution International License (CC BY 4.0).

http://creativecommons.org/licenses/by/4.0/

\begin{abstract}
The study seeks to report the clinical evolution of a patient who suffered an inadvertent subarachnoid puncture and evolved with a herpetic viral meningitis, confirmed by cerebrospinal fluid (CSF) analysis. Female, 48 years old, submitted to epidural anesthesia, for surgery, with median puncture, between L2-L3 and inadvertently accessed the subarachnoid space, with clear cerebrospinal fluid. The same puncture was used and the solution with $15 \mathrm{mg}$ hyperbaric bupivacaine and $80 \mathrm{mcg}$ morphine was administered. There was no complication during the entire surgical procedure. On the first postoperative day, she started complaining of headache and nausea, being treated with dipyrone, non-steroidal anti-inflammatory drugs (NSAIDs) and ondansetron. On the third day, she presented two episodes of seizure that ceased with the use of diazepam $10 \mathrm{mg}$. On the fifth day, the patient presented fever and seizures, which did not stop with medication, requiring sedation, orotracheal intubation and transfer to the intensive care unit. Lumbar puncture was performed for CSF analysis, which was positive for Herpes simplex type I. The patient was extubated, on the second day of hospitalization, maintained with a maintenance dose of phenytoin, sumatriptan, dipyrone, metoclopramide and acyclovir $500 \mathrm{mg}$, remaining intravenous, without presenting new seizures for 45 days, when she was discharged. After 30 days, he returned for a review consultation without making a complaint.
\end{abstract}

\section{Keywords}

Headache, Opioids, Herpes Simplex, Dural Puncture 


\section{Introduction}

Inadvertent dural puncture (IDP) during epidural puncture (EP) is a complication that occurs in approximately $0.91 \%$ of EP's [1]. In certain cases, inadvertent subarachnoid injection can happen, when the dura mater (DM) perforation is not recognized, which can generate important sequelae or even lead to potentially fatal consequences [2].

Certain complications of lumbar punctures are well known and documented, such as: headache, transient neurological disorders, subdural hematoma, abscesses, meningitis and encephalitis [3].

Meningitis as a consequence of inadvertent DM puncture is a rare complication; however, it is potentially fatal and can occur due to bacterial, viral or chemical causes [4]. Two important issues for the occurrence of meningitis after lumbar puncture are worth mentioning: violations of aseptic techniques or hematogenous propagation in the presence of asymptomatic bacteremia. Primary contamination of anesthetic equipment and agents as a cause is less likely [5].

Viral meningitis is more common than bacterial meningitis and tends to be less severe. The clinical course of viral meningitis is generally self-limited and with complete recovery within 7 to 10 days. It is known that the exact incidence of viral meningitis is underestimated due to inadequate reporting practices [6] [7]. Herpes simplex encephalitis (HSE) is the most common cause of acute viral encephalitis in the United States [8]. Up to two-thirds are thought to be due to virus reactivation rather than a primary infection, as $90 \%$ of the population is infected with herpes simplex virus (HSV) [8].

In the current literature, cases that relate the reactivation of the herpes simplex virus with mild or severe complications after lumbar puncture are rare [9]. As a result, the diagnosis of this clinical condition requires a high degree of suspicion, which can hinder early diagnosis, as well as effective treatment, which can be decisive in preventing serious complications [10].

The real reasons why patients with herpes simplex may present viral reactivation are not fully understood. Some studies question whether the use of opioids can be related to this event [11] [12] [13].

The objective of this study is to report the clinical evolution of a patient who suffered an inadvertent spinal puncture during epidural anesthesia, with a subsequent picture of herpetic viral meningitis, confirmed by CSF analysis, in addition to discussing its main differential diagnoses and, above all, highlight the relevant aspects of clinical propaedeutics that make specialist assessment necessary.

The free and informed consent form was obtained from the patient and the ethical questions of research in human beings were met.

\section{Case Report}

A female 48 years old, classified by the American Society of Anesthesiologists (ASA), with physical status ASA P1, candidate for Total Abdominal Hysterect- 
omy (TAH) surgery. Monitoring consisted of: continuous cardiac cardioscopy; non-invasive pressure; pulse oximetry and capnography. Venoclysis was performed on the left upper limb, with an $18 \mathrm{G}$ venous catheter, and intravenous sedation was performed with titrated doses of $4 \mathrm{mg}$ midazolam and $50 \mathrm{mcg}$ fentanyl. The patient was placed in the left lateral decubitus position, and asepsis and antisepsis were performed with 70\% alcohol, in addition to local anesthesia of the skin and subcuta/neous tissue with $2 \%$ lidocaine. Lumbar puncture was performed, via median, between L2-L3, with an 18G Tuohy needle and inadvertently accessed the subarachnoid space, with clear CSF exit. The same puncture was used and the solution with $15 \mathrm{mg}$ hyperbaric bupivacaine and $80 \mathrm{mcg}$ morphine was administered. Pre-oxygenation with $100 \% \mathrm{O}_{2}$ was performed for 5 minutes and then the following drugs were infused intravenously for anesthetic induction: lidocaine $60 \mathrm{mg}$; fentanyl $150 \mathrm{mcg}$; propofol $120 \mathrm{mg}$ and rocuronium $50 \mathrm{mg}$. Intubation was performed with a 7.5 cuffed tube, without complications.

During the surgical procedure, which lasted 250 minutes, there were no complications or hemodynamic instability. The patient was extubated at the end of the procedure and sent to the post-anesthetic recovery room (POR), with 9/10 points on the Aldrete-Kroulik scale, and was discharged to the ward.

On the first postoperative day, she complained of headache, nuchalgia and nausea, being treated with dipyrone, non-steroidal anti-inflammatory drugs and ondansetron

On the third postoperative day, the patient presented two episodes of convulsive crisis, which stopped with the use of diazepam $10 \mathrm{mg}$, in addition to maintenance with phenytoin $100 \mathrm{mg}$ every 8 hours, intravenously. A cranial tomography was performed, in which no acute ischemic or hemorrhagic alterations were evidenced.

On the fifth postoperative day, the patient developed fever and new convulsive crises, which did not stop with medication, requiring sedation, orotracheal intubation, as well as transfer to the intensive care unit (ICU). She was evaluated by the neurologist, and in the physical examination, the presence of neck stiffness and hyperreflexia was evidenced, and the diagnostic hypothesis of subarachnoid hemorrhage was raised. New imaging tests were requested, in addition to a blood count, which revealed the presence of 16,000 leukocytes and $6 \%$ rods. Due to the worsening of the clinical picture, a new lumbar puncture was performed for CSF analysis, with a xanthochromatic appearance, in addition to an increase in total protein $(149 \mathrm{mg} / \mathrm{dL})$, glucose $(91 \mathrm{mg} / \mathrm{dL})$, numerous red blood cells, rare white blood cells and polymerase chain reaction (rT-PCR) test positive for Herpes simplex type I. Nasal, rectal swab and urine culture negative.

The patient was extubated on the second day of admission to the ICU, maintained on a maintenance dose of phenytoin, sumatriptan, dipyrone, metoclopramide and acyclovir $500 \mathrm{mg}$ intravenously, with no further seizures.

The patient remained hospitalized for 45 days, with no further seizures, and was discharged from the hospital. She returned after 30 days for the follow-up 
visit without any complaints, neurological symptoms or cutaneous manifestations.

\section{Discussion}

In this case report, the patient was submitted to an TAH, through general anesthesia associated with spinal anesthesia, presenting headache after inadvertent dural puncture ( $24 \mathrm{~h}$ after puncture), followed by nucalgia and vomiting. Symptoms could be, initially, related to a picture of headache after dural puncture [14] [15]. Post-dural puncture headache (PDPH) is a not-so-rare event arising from neuraxial anesthesia, occurring in approximately $1.5 \%-3.6 \%$ of cases, reaching approximately $70 \%$ in cases related to inadvertent dural perforation - mater (Tuohy's epidural needle) being more frequently observed in young and pregnant patients, who received neuraxial anesthesia [16]. Its main differential diagnoses are listed in Table 1.

The typical location of the headache is usually bifrontal and/or occipital, being described as intense, disturbing and debilitating, which increases in the vertical position and decreases or disappears completely in the supine position [17].

$\mathrm{PDPH}$ is related to several factors, such as: age, female gender, number of puncture attempts and use of larger gauge needles. Even so, its etiology has not been completely elucidated, with the most accepted explanation being the caudal displacement of the brain and the traction of the meninges due to low CSF pressure related to the loss of CSF through the puncture orifice. Conventional treatment also differs in the literature, with relative rest being indicated for pain of mild to moderate intensity, in addition to clinical treatment with analgesics, anti-inflammatory drugs, adjuvant drugs and caffeine. In severe pain, it is suggested to try clinical treatment and, in refractory cases, to indicate blocks, such as sphenopalatine and epidural blood patch [18] [19].

According to the criteria presented in the third edition of the International Classification of Headache Disorders (ICHD), published by the International Headache Society, in 2018, PDPH is characterized as a headache that occurs within 5 days after a lumbar puncture, caused by a leakage of spinal cerebrospinal fluid (CSF) through dural puncture. In addition, it may be accompanied by neck stiffness, tinnitus, hearing alterations, photophobia and/or nausea, as well as the postural character related to pain [2]-[15].

In this case, although some characteristics presented are common to the PDPH clinic, some of them drew attention Table 2, such as the fact that the

Table 1. Differential diagnoses of post-dural puncture headache.

Primary headaches (tension headache and migraine);
Pneumoencephalon;
Meningitis;
Expansive lesions (primary tumors, metastatic lesions, bruises and abscesses);
Venous sinus thrombosis.


Table 2. Characteristics of headaches that need more attention.

\begin{tabular}{l}
\hline Severe headache with sudden onset; \\
Spontaneous report of the worst headache of life; \\
Headache in patients in extreme age; \\
Headache in cancer/immunosuppressed patients; \\
Changes in neurological examination (eg, papilledema; neck stiffness and \\
sub hyaloid hemorrhage); \\
Weight loss; seizures; behavioral and level of consciousness changes).
\end{tabular}

patient complains of an intense headache that is not related to the postural component. Therefore, the evaluation by the neurologist was suggested, who, during the patient's investigation, identified changes in the neurological examination and requested imaging tests, suggesting a possible situation of subarachnoid hemorrhage, considering the performance of a lumbar puncture with CSF output from xanthochromatic appearance. However, cranial Computed Tomography (CT) and Nuclear Magnetic Resonance (NMR) did not show signs of bleeding. The CSF analysis was positive for Herpes simplex type I (HSV) by polymerase chain reaction (rt-PCR), which determined the diagnosis of herpetic viral meningitis, allowing the beginning of effective treatment early.

The early diagnosis of herpes simplex type I meningitis after inadvertent subarachnoid puncture is complex and challenging, as, as reported in this case, the signs and symptoms presented by the patient can be very similar to other conditions and pathologies [20].

In this sense, the etiological diagnosis becomes imperative for the institution of adequate treatment. In the case of herpes simplex virus type I infection, it is very important, because encephalitis caused by this agent has a mortality rate of $70 \%$, if not treated. When treated, this percentage can be reduced to $19 \%$, but even so, half will have sequelae [2].

Crone and James et al., reported the association between the use of morphine and the reactivation of herpes simplex type I, after its epidural administration in obstetric patients [11] [12]. Hoesni et al. reported a case of herpetic meningitis in an obstetric patient after inadvertent dural puncture [9].

Some studies report a potential immunosuppressive role of morphine in humans (acute and chronic use) [21] [22] [23] [24] [25]. One of these studies also demonstrated a tendency to reduce IFN $-\gamma$ by CD $8+$ cells $24 \mathrm{~h}$ after epidural puncture [13].

It is known that HSV-1 latency occurs when histone deacetylases maintain chromatin in an inactive state, allowing the IFN- $\gamma$ produced by Natural Killer cells and CD8+ T cells to exert its antiviral effect [26]. Thus, it is assumed that, with the potential decrease in IFN- $\gamma$ expression exerted by morphine, there would be a greater chance of virus reactivation. Other possible causes listed in the literature for HSV reactivation include: direct damage to nervous structures and the use of systemic corticosteroids [27]. 


\section{Conclusion}

This case is an event little described in the literature, in which it was postulated that the inadvertent puncture of the subarachnoid space, with injection of hydrophilic opioid (morphine), could be related to the reactivation of dormant HSV-1 in the patient, which led to the condition of reported herpetic meningitis. Further studies should be carried out to clarify the role of morphine in HSV reactivation in patients with dormant herpes. It is necessary to be aware of the warning signs to early identify possible threatening etiologies, which should receive additional evaluation and specialist follow-up. In these cases, early diagnosis, as well as the institution of individualized treatment, is essential for improving outcomes.

\section{Conflicts of Interest}

The authors declare no conflicts of interest regarding the publication of this paper.

\section{References}

[1] Sprigge, J.S. and Harper, S.J. (2008) Accidental Dural Puncture and Post Dural Puncture Headache in Obstetric Anaesthesia: Presentation and Management: A 23-Year Survey in a District General Hospital. Anaesthesia, 63, 36-43. https://doi.org/10.1111/j.1365-2044.2007.05285.x

[2] Amorim, J.A., et al. (2010) Intracranial Subdural Hematoma Post-Spinal Anesthesia: Report of Two Cases and Review of 33 Cases in the Literature. Revista Brasileira de Anestesiologia, 60, 624-629. https://doi.org/10.1016/S0034-7094(10)70077-5

[3] Bisinotto, F.M.B., et al. (2012) Hematoma Subdural Intracraniano: Uma rara complicação após raquianestesia: Relato de caso. Revista Brasileira de Anestesiologia, 62, 92-95. https://doi.org/10.1590/S0034-70942012000100012

[4] Govardhane, B.T., Jambotkar, T.C., Magar, J.S., et al. (2015) Meningitis Following Spinal Anesthesia. Medical Journal of Dr. DY Patil University, 8, 513. https://doi.org/10.4103/0975-2870.160818

[5] Johnson, K.S. and Sexton, D.J. (2013) Lumbar Puncture: Technique, Indications, Contraindications, and Complications in Adults.

[6] Zueter, A.M. and Zaiter, A. (2015) Infectious Meningitis. Clinical Microbiology Newsletter, 37, 43-51. https://doi.org/10.1016/j.clinmicnews.2015.02.004

[7] Bartt, R. (2012) Acute Bacterial and Viral Meningitis. CONTINUUM: Lifelong Learning in Neurology, 18, 1255-1270. https://doi.org/10.1212/01.CON.0000423846.40147.4f

[8] Harrison, K.S., Zhu, L., Thunuguntla, P., et al. (2020) Herpes Simplex Virus 1 Regulates $\beta$-Catenin Expression in TG Neurons during the Latency-Reactivation Cycle. PLoS ONE, 15, e0230870. https://doi.org/10.1371/journal.pone.0230870

[9] Hoesni, S., et al. (2010) Herpes Simplex Meningitis after Accidental Dural Puncture during Epidural Analgesia for Labour. International Journal of Obstetric Anesthesia, 19, 466-467. https://doi.org/10.1016/j.ijoa.2010.07.022

[10] Kneen, R., Michael, B.D., Menson, E., et al. (2012) Management of Suspected Viral Encephalitis in Children-Association of British Neurologists and British Paediatric Allergy, Immunology and Infection Group National Guidelines. Journal of Infection, 64, 449-477. https://doi.org/10.1016/j.jinf.2011.11.013 
[11] Crone, L.A., et al. (1990) Herpes Labialis in Parturients Receiving Epidural Morphine Following Cesarean Section. Anesthesiology, 73, 208-213. https://doi.org/10.1097/00000542-199008000-00003

[12] James, C.F. (1996) Recurrence of Herpes Simplex Virus Blepharitis after Cesarean Section and Epidural Morphine. Anesthesia \& Analgesia, 82, 1094-1096.

https://doi.org/10.1213/00000539-199605000-00042

[13] Chen, S.H., et al. (2019) Effects of Systemic and Neuraxial Morphine on the Immune System. Medicine (Baltimore), 98, e15375. https://doi.org/10.1097/MD.0000000000015375

[14] Lim, G., et al. (2016) Subdural Hematoma Associated with Epidural Analgesia: A Case Series. Regional Anesthesia \& Pain Medicine, 41, 628-631. https://doi.org/10.1097/AAP.0000000000000455

[15] Arnold, M. (2018) Headache Classification Committee of the International Headache Society (ihs) the International Classification of Headache Disorders. Cephalalgia, 38, 1-211. https://doi.org/10.1177/0333102417738202

[16] Sachs, A. and Smiley, R. (2014) Post-Dural Puncture Headache: The Worst Common Complication in Obstetric Anesthesia. Seminars in Perinatology, 38, 386-394. https://doi.org/10.1053/j.semperi.2014.07.007

[17] TurnbulL, D.K. and Shepherd, D.B. (2003) Post-Dural Puncture Headache: Pathogenesis, Prevention and Treatment. British Journal of Anaesthesia, 91, 718-729. https://doi.org/10.1093/bja/aeg231

[18] Leibold, R.A., et al. (1993) Post-Dural-Puncture Headache: Characteristics, Management, and Prevention. Annals of Emergency Medicine, 22, 1863-1870. https://doi.org/10.1016/S0196-0644(05)80416-0

[19] Puthenveettil, N., Rajan, S., Mohan, A., et al. (2018) Sphenopalatine Ganglion Block for Treatment of Post-Dural Puncture Headache in Obstetric Patients: An Observational Study. Indian Journal of Anaesthesia, 62, 972.

[20] Logan, S.A.E. and Macmahon, E. (2008) Viral Meningitis. BMJ, 336, 36-40. https://doi.org/10.1136/bmj.39409.673657.AE

[21] Han, C., Lei, D., Liu, L., et al. (2020) Morphine Induces the Differentiation of T Helper Cells to Th2 Effector Cells via the PKC- $\theta$-GATA3 Pathway. International Immunopharmacology, 80, Article ID: 106133. https://doi.org/10.1016/j.intimp.2019.106133

[22] Acharya, A., Olwenyi, O.A., Thurman, M., et al. (2021) Chronic Morphine Administration Differentially Modulates Viral Reservoirs in a Simian Immunodeficiency Virus SIVmac251-Infected Rhesus Macaque Model. Journal of Virology, 95, e01657-20. https://doi.org/10.1128/JVI.01657-20

[23] Maher, D.P., Walia, D., Heller N.M., et al. (2020) Morphine Decreases the Function of Primary Human Natural Killer Cells by Both TLR4 and Opioid Receptor Signaling. Brain, Behavior, and Immunity, 83, 298-302. https://doi.org/10.1016/j.bbi.2019.10.011

[24] Longhini, F., Bruni, A., Garofalo, E., et al. (2020) Anesthetic Strategies in Oncological Surgery: Not Only a Simple Sleep, But Also Impact on Immunosuppression and cancer Recurrence. Cancer Management and Research, 12, 931. https://doi.org/10.2147/CMAR.S237224

[25] Hudzik, B., Nowak, J. and Zubelewicz-Szkodzinska, B. (2020) Consideration of Immunomodulatory Actions of Morphine in COVID-19-Short Report. European Review for Medical and Pharmacological Sciences, 24, 13062-13064. 
[26] Bigley, N.J. (2014) Complexity of Interferon- $\gamma$ Interactions with HSV-1. Frontiers in Immunology, 5, 15. https://doi.org/10.3389/fimmu.2014.00015

[27] McLaughlin, D.C., Achey, R.L., Geertman, R., et al. (2019) Herpes Simplex Reactivation Following Neurosurgery: Case Report and Review of the Literature. Neurosurgical Focus, 47, E9. https://doi.org/10.3171/2019.5.FOCUS19281 